TITELTHEMA HOCHVOLT- UND 48-V-SYSTEME

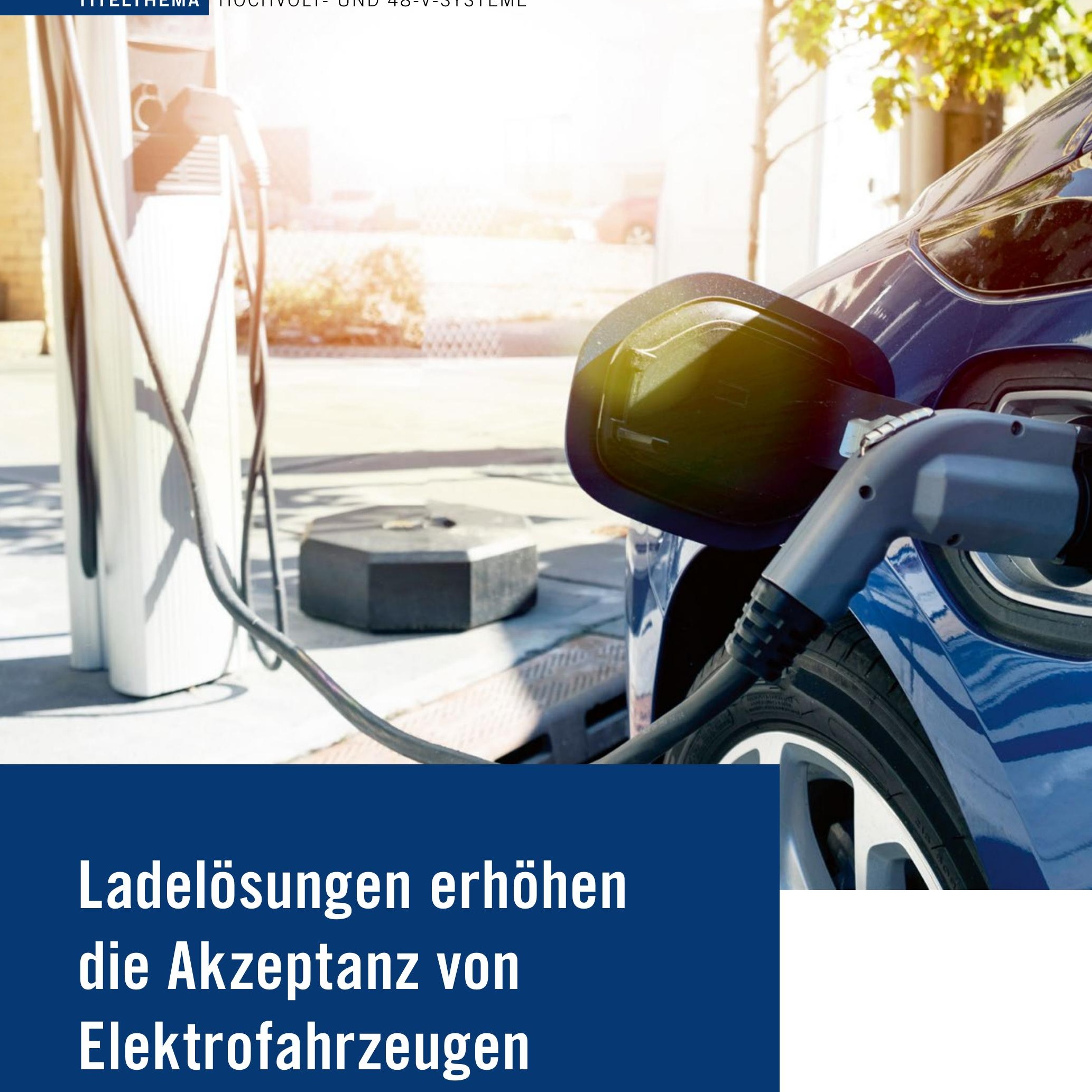

Die Verfügbarkeit einer schnellen DC-Lade-Infrastruktur ist ein wesentliches Kriterium für den Erfolg von batteriebetriebenen Elektrofahrzeugen. Infineon beschreibt, wie ausreichende Möglichkeiten für das Schnellladen mit akzeptabler Dauer geschaffen werden können. 
Darüber hinaus gibt es viele Länder, die als Reaktion auf Covid-19-Förderprogramme für die Wirtschaft aufgelegt haben. Häufig profitieren von diesen Regierungsprogrammen vor allem Elektrofahrzeuge (BEVs), entweder direkt über entsprechende Verkaufsanreize, oder indirekt über Infrastrukturausgaben für Ladestationen. Vor diesem Hintergrund könnte die Pandemie die Revolution der E-Mobilität sogar noch beschleunigen. Die Automobilhersteller investieren Milliarden in die Entwicklung von Elektro- und Hybridfahrzeugen und entsprechende Verkaufsanstrengungen. Das Henne-Ei-Problem scheint sich damit langsam aufzulösen, denn auch für das Laden der BEVs gibt es inzwischen nutzerorientierte Lösungen.

\section{DERZEITIGE LADEOPTIONEN}

Für das Aufladen der Batterie zuhause unterstützen die meisten Fahrzeuge das Laden über eine haushaltsübliche einphasige Wechselspannungsversorgung (AC). Das ermöglicht eine Ladung über Nacht. Die Lösungen reichen von einem einfachen Kabel, um das Fahrzeug an die Steckdose anzuschließen, über solche mit integrierten Kontroll- und Schutzelementen (IC-CPD) bis hin zu komplexeren Ladeeinrichtungen mit Wandmontage. Diese ermöglichen zum Teil auch die Kommunikation zwischen dem Fahrzeug und der Stromversorgung sowie die Erdung und weitere Schutzfunktionen.

Die Batterien selbst benötigen eine Gleichspannungsversorgung (DC) und damit eine AC/DC-Umwandlung mit entsprechender Ladeelektronik im Fahrzeug. Das bedeutet wiederum, dass das Fahrzeug über eine Ladefunktion verfügen muss, die alle Aspekte wie Kühlung, Effizienz oder Gewicht berücksichtigt alles Faktoren, welche die Ladeleistung und damit die Ladegeschwindigkeit limitieren. Vor diesem Hintergrund ist die Entwicklung eines universellen externen DC-Ladesystems erforderlich.

\section{ANSÄTZE FÜR EINE SCHNELLE GLEICHSPANNUNGSLADUNG}

Ein typisches 22-kW-DC-Ladesystem kann in rund 120 min das Fahrzeug für eine Reichweite von zusätzlichen 200 km aufladen. Das ist mehr als ausreichend für das Laden während der Arbeit. Um aber die Ladezeit für die 200-km-Reich-
AUTOREN

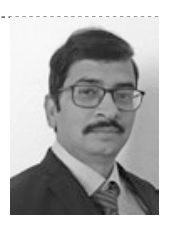

Pradip Chatterjee ist Application Engineer EV Charging bei Infineon Technologies in Warstein.

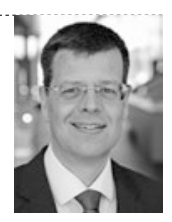

Markus Hermwille ist Director Business

Development bei Infineon Technologies in Neubiberg.

weite auf nur $16 \mathrm{~min}$ zu verkürzen, ist bereits eine DC-Ladestation mit $150 \mathrm{~kW}$ erforderlich. Mit $350 \mathrm{~kW}$ Leistung reduziert sich der Ladevorgang auf nur noch 7 min, und man kommt so in die Nähe des Zeitraums, der für das Auftanken konventioneller Fahrzeuge benötigt wird. Diese Zahlenbeispiele setzen natürlich voraus, dass die Batterie diese Laderaten auch unterstützen kann. Neben der Möglichkeit, das Elektrofahrzeug schnell zu laden, wünschen sich Kunden darüber hinaus ein standardisiertes Verfahren wie an der Zapfsäule, egal wo die Batterie geladen wird.

In Europa treibt die Organisation CharIN e.V. die Entwicklung und Promotion des Combined Charging System (CCS) voran. Die Spezifikationen definieren den Ladestecker, die Ladesequenz und auch die Datenkommunikation. Andere Regionen wie Japan und China haben mit CHAdeMO beziehungsweise $\mathrm{GB} / \mathrm{T}$ vergleichbare Organisationen, während Tesla ein eigenes proprietäres System nutzt. Die CharIN-Spezifikationen unterstützen sowohl das AC- als auch das DC-Laden über ihre Steckerimplementierung. Außerdem ist ein maximaler Konstantstromausgang von 500 A bei 700 VDC, mit Unterstützung bis zu 920 VDC definiert. Derzeit ist der Wirkungsgrad mit $95 \%$ angegeben, er soll aber künftig auf $98 \%$ steigen. Hier ist anzumerken, dass ein um $1 \%$ geringerer Wirkungsauf $59 \mathrm{~g} \mathrm{CO}_{2} / \mathrm{km}$ gesenkt. 


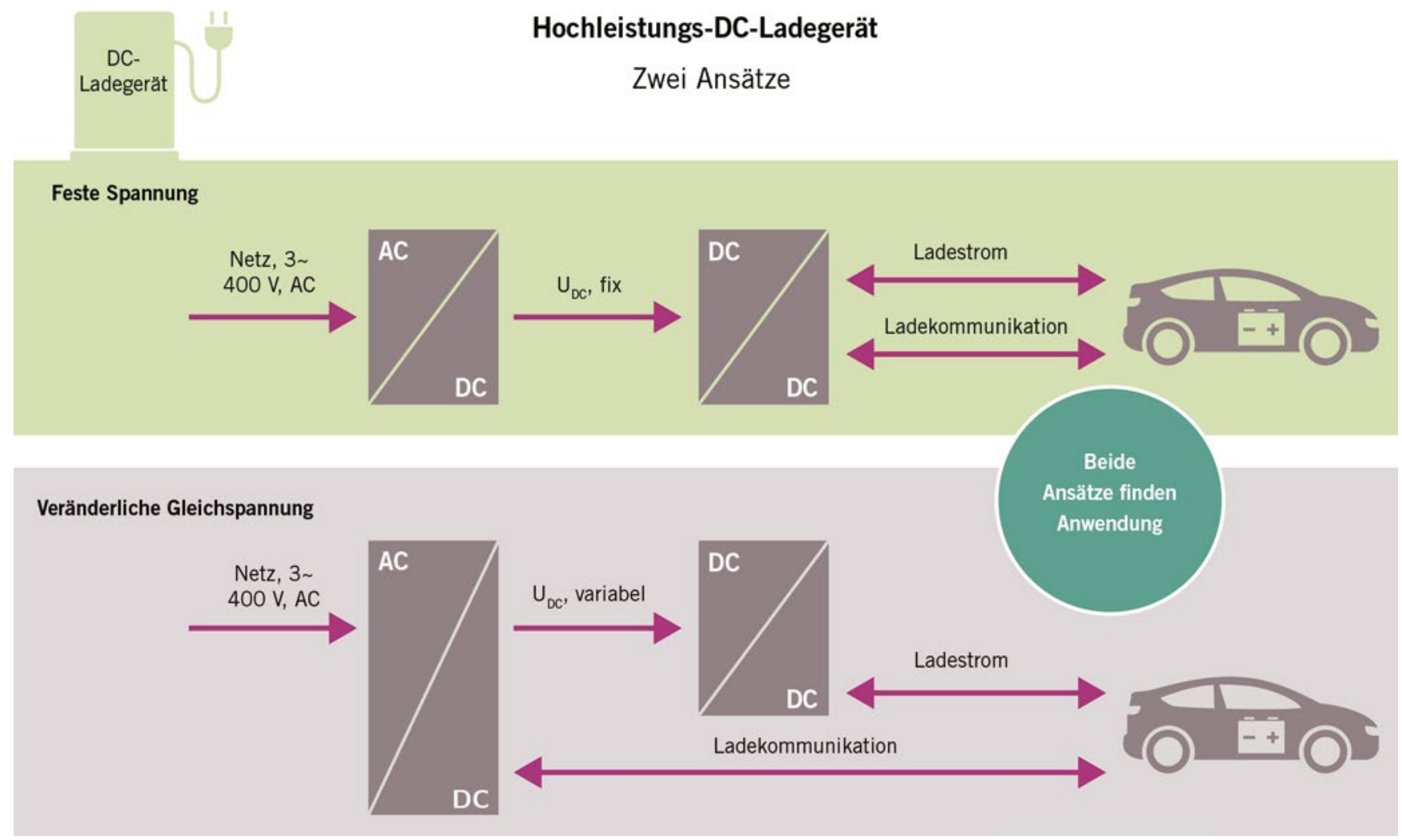

BILD 1 Blockdiagramme für zwei mögliche Architekturen für Hochleistungs-DC-Ladesysteme (@ Infineon Technologies)

grad bei einem 150-kW-Ladesystem immerhin $1,5 \mathrm{~kW}$ entspricht. Daher gilt es, die Verluste bei einem schnellen DC-Ladesystem auf ein absolutes Minimum zu reduzieren.

\section{ARCHITEKTUREN FÜR SCHNELLE DC-LADESYSTEME}

Das Design von DC-Ladesystemen für das Schnellladen basiert typischerweise auf zwei verschiedenen Ansätzen. Die erste Architektur konvertiert eine dreiphasige AC-Versorgung in einen variablen DC-Ausgang, der einen DC/DCWandler speist. Die genaue DC-Spannung wird mittels Kommunikation mit dem zu ladenden Fahrzeug ermittelt. Bei dem alternativen Ansatz wird die anliegende AC-Spannung in eine fixierte DCSpannung konvertiert, wobei dann der DC/DC-Wandler die Ausgangsspannung für die Fahrzeugbatterie anpasst, BILD 1.

Beide Architekturen haben Vor- und Nachteile, sodass letztlich die Systemanforderungen den optimalen Ansatz bestimmen. Derartige Hochleistungslösungen werden keine monolithische Umsetzung nutzen. Vielmehr wird die gewünschte Ausgangsleistung durch Kombination mehrerer Lade-Subsysteme erreicht werden, die jeweils Leistungen von 15 bis $60 \mathrm{~kW}$ beitragen. Hierbei ist es wichtig, den Kühlaufwand möglichst gering zu halten sowie eine hohe Leistungsdichte und möglichst geringe Abmessungen zu erreichen.

Die Effizienz eines Designs beginnt am Frontend mit der AC/DC-Umsetzungsstufe. Die Implementierung der Blindleistungskompensation nutzt üblicherweise eine Topologie mit ViennaGleichrichter. Der Einsatz von aktiven 600-V-Bauelementen mit den Vorteilen des 3-Level-Gleichrichters ermöglicht eine optimale Balance zwischen Kosten und Leistungsfähigkeit. Mit der Verfügbarkeit von Hochvolt-Bauelementen auf Basis von Siliziumkarbid (SiC) kann aber auch eine AC/DC-Konvertierungsstufe mit gängiger 2-Level-PWM-Topologie in Bereichen von $50 \mathrm{~kW}$ und höher eingesetzt werden. Mit beiden Ansätzen kann bei einem sinusförmigen Eingangsstrom eine kontrollierte Ausgangsspannung mit einem Leistungsfaktor von über 0,95, einer Total Harmonic Distortion (THD) von weniger als $5 \%$ und einem Wir- kungsgrad mit über $97 \%$ erreicht werden. In Anwendungen, in denen eine Isolierung gegenüber dem Stromnetz mit einem Umsetzer mittlerer Spannung möglich ist, können auch MultipulsGleichrichter-Topologien mit einer Diode und einem Thyristor genutzt werden. Diese sind einfach einzusetzen, zuverlässig und bieten eine höhere Effizienz. In der DC/DC-Stufe werden oft resonante Topologien aufgrund ihrer Effizienz und galvanischen Isolierung bevorzugt. Derartige Designs erfüllen die Anforderungen nach hoher Leistungsdichte mit kompakten Abmessungen, während das spannungslose Schalten von Schalttransistoren die Schaltverluste reduziert und so die Systemeffizienz erhöht. Die Vollbrückentopologie mit Phasenverschiebung mit SiC-Bauelementen stellt eine alternative Lösung für isolierte Designs dar. In Architekturen mit Isolierung zum Stromnetz sind Multiinterleaved-Abwärtswandler (Buck-Konverter) die bevorzugte DC/DC-Topologie. Der Vorteil hierbei ist, dass die Last über alle Phasen verteilt werden kann sowie eine reduzierte Restwelligkeit (Rippel) und kleinere Filter. Verbunden ist das 
Typische Lösungen für Ladegeräte mit 30 bis 150 kW: diskrete Bauelemente

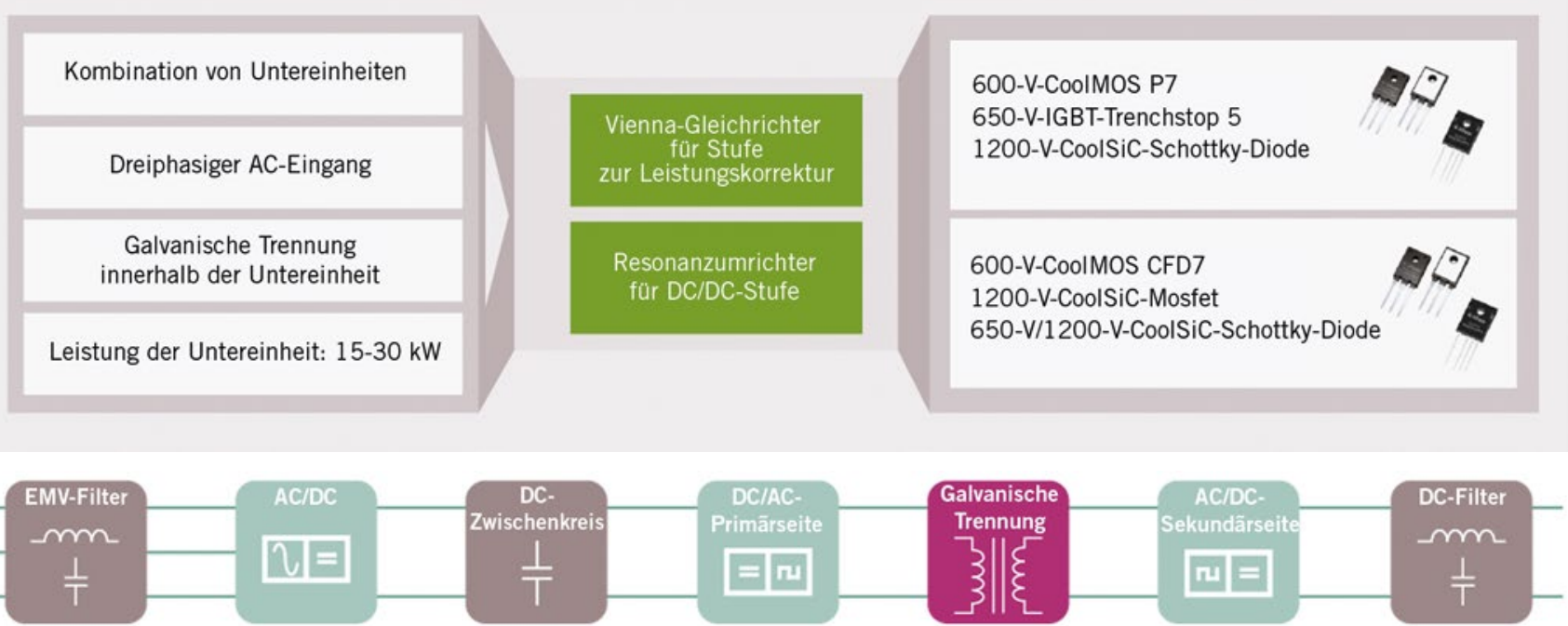

BILD 2 Typische Topologie für ein Ladesystem auf Basis von diskreten Bauelementen () Infineon Technologies)

allerdings auch mit dem Nachteil, dass mehr Bauteile benötigt werden.

Im Leistungsbereich von 15 bis $30 \mathrm{~kW}$ werden die Subsysteme am besten mit diskreten Komponenten, BILD 2 implementiert. So bietet der Einsatz von Trenchstop-5-IGBTs zusammen mit CoolSiC-Schottky-Dioden in einem Vienna-Gleichrichter eine gute Kombination für kostensensitive Anwendungen. Indem man die IGBTs durch CoolMOS-P7-SJ-Mosfets ersetzt, kann die Effizienz nochmals geringfügig gesteigert werden. Für den DC/DC-Konverter erreicht man mit einem resonanten Umsetzer und CoolMOS-CFD7-Mosfets einen guten Wirkungsgrad. Mosfets aus dem CoolSiC-Portfolio empfehlen sich, wenn höchste Effizienz das Ziel ist.
Trotz der sehr guten Wirkungsgrade, die Leistungsumrichter heute erreichen können, entspricht ein Wirkungsgradverlust von nur $1 \%$ einer Verlustleistung von 3,5 kW als Wärme, die im System abgeführt werden muss.

Bei 15- bis 22-kW-Ladegeräten ist der Ansatz eines zwangsluftgekühlten Systems sehr verbreitet. Ein geräuscharmer Lüfter, weniger als $60 \mathrm{~dB}$, mit geeigneter Luftführung und Staubfilter findet in den aktuellen Installationen breite Akzeptanz. Mit der Notwendigkeit eines flüssigkeitsgekühlten Kabels für DC-Ladesysteme ab 120 kW gewinnt das Konzept des flüssigkeitsgekühlten Umrichters immer mehr an Bedeutung. Die Herausforderung besteht darin, dass viele flüssige Kühlmittel in puncto Entflammbarkeit, Zerset- zung, Korrosion und Toxizität Probleme bereiten. Heute hat sich ein WasserGlykol-Gemisch als Kühlmittel sowohl für das Kabel als auch für den Stecker bewährt. Es wurden auch dielektrische Kühlmittel entwickelt, die erfolgreich bei leistungsstarken DC-Ladesystemen eingesetzt werden.

Dank der modernen Leistungstransistortechnologie, gekoppelt mit leistungsstarken System-Level-Controllern und fortschrittlichen magnetischen Materialien, können hocheffiziente $\mathrm{AC} / \mathrm{DC}$ Gleichrichterschaltungen leicht implementiert werden. Diese sind erforderlich, um eine sinusförmige Stromentnahme aus dem Netz mit Leistungsfaktorkorrektur, geringer harmonischer Verzerrung (THDi $\leq 5 \%$ ) und unabhängiger

\section{eMobility Measurement Technology Messtechnik für die Mobilität von Morgen

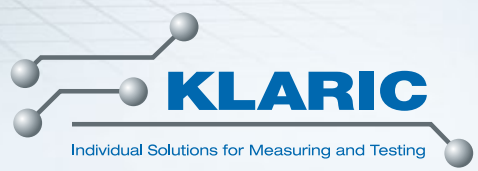


Typische Lösungen für Ladegeräte mit 50 bis 350 kW: Leistungsmodule

\begin{tabular}{|c|c|c|c|}
\hline Kombination von Untereinheiten & \multirow{2}{*}{$\begin{array}{l}\text { Aktive Front-End-Topologie } \\
\text { (AFE) mit Vienna- } \\
\text { Gleichrichter für } \\
\text { Stufe zur Leistungskorrektur }\end{array}$} & \multirow{2}{*}{$\begin{array}{l}\text { CooISiC-Easy-Modul } \\
\text { IGBT-EconoPack } \\
\text { IGBT-EconoDual } \\
\text { Gleichrichterdiodenmodul }\end{array}$} & \\
\hline Dreiphasiger Eingang & & & \\
\hline $\begin{array}{l}\text { Galvanische Trennung innerhalb } \\
\text { der Untereinheit }\end{array}$ & $\begin{array}{l}\text { Resonanzumrichter } \\
\text { (z. B. dreiphasig versetzt), } \\
\text { Abwärtswandler für }\end{array}$ & \multirow{2}{*}{$\begin{array}{l}\text { CoolSiC-Easy-Modul } \\
\text { IGBT-EconoPack } \\
\text { IGBT-EconoDual }\end{array}$} & \\
\hline Leistung der Untereinheit: $15-100 \mathrm{~kW}$ & & & \\
\hline
\end{tabular}

BILD 3 Typische Lösungen für Ladesysteme auf Basis von Leistungsmodulen (๑ Infineon Technologies)

Steuerung des Wirk- und Blindleistungsflusses bei gleichzeitig hoher Dynamik zu gewährleisten. Bei der Auswahl einer geeigneten Topologie für die Implementierung müssen die Ingenieure einige Entscheidungen treffen, die je nach Priorität von Kosten, Leistung und Leistungsdichte den uni- oder bidirektionalen Betrieb, die 2-Level- oder 3-Level-Topologie, Silizium- oder Siliziumkarbid-Mosfet-basierte Leistungshalbleiter betreffen.

Eine der am weitesten verbreiteten Topologien für den bidirektionalen Betrieb ist der sogenannte 2-Level-Voltage-Source-Converter (2L-VSC). Dieser besteht aus einer Anordnung von sechs Leistungsschaltern, typischerweise IGBTs oder Siliziumkarbid-Mosfets. Diese erzeugen zusammen mit einem Kondensator als Zwischenkreis eine Ausgangsspannung, die höher ist als die Spannungen in der Eingangsphase. Dabei kann entweder die sinusförmige Pulsbreitenmodulation (SPWM) oder die Raumzeigermodulation (SVM) verwenden werden. Mit einem CoolSiC-MosfetModul lassen sich kleinere Leistungsklassen von 6,6 bis $11 \mathrm{~kW}$ realisieren. Für höhere Leistungsklassen ab 22 kW bietet sich Halbbrückenlösungen mit einem Durchgangswiderstand von nur $2 \mathrm{~m} \Omega$ im 62-mm-Gehäuse, bis $6 \mathrm{~m} \Omega$ im Easy $2 \mathrm{~B}$ und bis $45 \mathrm{~m} \Omega$ im EasylB an.

Der dreiphasige, dreistufige ViennaGleichrichter wird in der Regel bevorzugt, wenn ein bidirektionaler Stromfluss nicht benötigt wird und der Schwerpunkt beim Design auf der Reduzierung der Schaltbeanspruchung und der EMIFilteranforderungen liegt. Ein symme- trischer Boost-PFC-Vienna-Gleichrichter kann mit Siliziumkarbid-MosfetBausteinen implementiert werden. Jedes Modul enthält zwei 1600-V-Gleichrichterdioden, zwei schnelle 1200-V-3-LevelDioden und zwei 1200-V/15-m $\Omega$-Siliziumkarbid-Mosfets. Das garantiert eine optimierte Lösung für ein kompaktes und verlustarmes Hochstrom-Design, BILD 3.

\section{ANSTEUERUNG, REGELUNG, KOMMUNIKATION UND SICHERHEIT}

Für die direkte Ansteuerung der Leistungsschalter werden in der Regel galvanisch isolierte Gatetreiber-ICs aus der EiceDriver-Familie eingesetzt. Diese eigenen sich für IGBTs der Trenchstop-5-Familie, CoolMOSP7-SJ-Mosfets oder Mosfets aus dem CoolSiC-Portfolio. Die Regelung von Leistungsstufen erfolgt üblicherweise mit einem Mikrocontroller. Controller aus der XMC4000-Serie bieten dafür flexible AC/DC-Wandler zusammen mit hochkonfigurierbaren Timern und PWM-Peripherie, um die Regelschleife zu implementieren. Eine CANSchnittstelle ermöglicht die Kommunikation unter den Teileinheiten und sorgt für die Übermittlung der Daten der verschiedenen Batterietypen. Serviceabrechnungen sowie die Authentifizierung von Software-Updates und HardwareUpgrades können über das HardwareSecurity-Modul (HSM) in den AurixMikrocontrollern sicher abgewickelt werden. Die Familie hat sich in zahlreichen sicherheitsrelevanten Anwendungen der Automobilindustrie bereits bewährt. Die Authentifizierung beim
Austausch von Subsystemen kann mit Security-Chips wie dem OptigaTrust-B gegenüber Manipulation geschützt werden. Für umfassendere Schutzmaßnahmen stehen darüber hinaus die Optiga-TPM-Trusted-Platform-Module zur Verfügung.

\section{ZUSAMMENFASSUNG}

Die Verfügbarkeit einer schnellen DCLadeinfrastruktur ist ein wesentliches Kriterium für den Erfolg von batteriebetriebenen Elektrofahrzeugen. Ohne ausreichende Möglichkeiten für das Schnellladen mit akzeptabler Dauer werden BEVs auf die Nutzung für kleinere Entfernungen begrenzt bleiben. Die Voraussetzungen wie die Spezifizierung von Ladeeinrichtungen und Steckverbindern wurden geschaffen. Darüber hinaus stehen auch innovative Halbleiterlösungen bereit. Diese reichen von Silizium-Leistungsbauelementen bis zu SiC-Varianten, die auch gut bei höheren Schaltfrequenzen genutzt werden können - für eine noch effizientere Leistungsumsetzung. Damit werden Ladesysteme effizient und zuverlässig. In Kombination mit entsprechenden Mikrocontrollern sowie ausgefeilten Authentifizierungs- und Security-Lösungen kann die DC-Ladeinfrastruktur für zukünftige Verkehrsanforderungen realisiert werden. 